EUROPEAN JOURNAL OF PURE AND APPLIED MATHEMATICS

Vol. 14, No. 4, 2021, 1132-1147

ISSN 1307-5543 - ejpam.com

Published by New York Business Global

\title{
Definite integral of a hyperbolic quotient function expressed in terms of the Lerch function
}

\author{
Robert Reynolds $^{1, *}$, Allan Stauffer ${ }^{1}$ \\ 1 Department of Mathematics and Statistics, Faculty of Science, York University, Toronto, \\ Ontario, Canada, MзJ1P3
}

\begin{abstract}
In applied sciences it is always useful to improve the catalogue of definite integrals available in tables. This present paper is a compendium of definite integrals involving a hyperbolic quotient function expressed in terms of the Lerch function. A substantial portion of the results are new.
\end{abstract}

2020 Mathematics Subject Classifications: 30E20, 33-01, 33-03, 33-04, 33-33B, 33E20, 33E33

Key Words and Phrases: Entries in Gradshteyn and Ryzhik, Mellin transform, Hyperbolic function, Definite integral, Catalan's constant

\section{Significance Statement}

The works of Bonderson and Gorda $[2,4]$ are concerned with some very interesting topics in particle and plasma physics. Within these articles the authors used some mathematical formula from the book of Gradshteyn and Ryzhik [8]. In this paper the authors provided a formal derivation for the formulae used in $[2,4]$ along with deriving generalized forms for some known and new integrals. The definite integrals derived in this work are useful in applications, in particular in perturbation analysis of solitons and plasma physics $[1,3,5,6,9,14]$.

The derived integral formula in this present work is expressed in terms of Lerch function. The Lerch function being a special function has the fundamental property of analytic continuation, which enables us to widen the range of evaluation for the parameters involved. We provide formal derivations of some formula in the books of [8] and [12] not previously published to the best of our knowledge.

* Corresponding author.

DOI: https://doi.org/10.29020/nybg.ejpam.v14i4.4029

Email addresses: milver@my.yorku.ca (R. Reynolds), stauffer@yorku.ca (A. Stauffer) 


\section{Introduction}

The definite integral derived in this manuscript is given by

$$
\int_{0}^{\infty} \operatorname{csch}^{2}(c x)\left(e^{-2 m x}(\log (a)-2 x)^{k}+e^{2 m x}(\log (a)+2 x)^{k}-2 \log ^{k}(a)\right) d x
$$

where the parameters $k, a$ are general complex numbers and $|R e(c)|>m$. The derivation of the definite integral follows the method used by us in [13] which involves Cauchy's integral formula. The generalized Cauchy's integral formula is given by

$$
\frac{y^{k}}{\Gamma(k+1)}=\frac{1}{2 \pi i} \int_{C} \frac{e^{w y}}{w^{k+1}} d w .
$$

where $\mathrm{C}$ is in general an open contour in the complex plane where the theorem in [13] gives the result. This method involves using a form of equation (2) then multiply both sides by a different function, then take a definite integral of both sides. This yields a definite integral in terms of a contour integral. A second contour integral is derived by multiplying equation (2) by a function and performing some substitutions so that the contour integrals are the same.

\section{Definite integral of the contour integral}

We use the method in [13]. We will derive three contour integral representations and add them such that we get an equivalent form for the infinite sum. Deriving the first contour we use equation (2) and replace $y$ by $\log (a)$ and multiply by $-\frac{1}{2} \operatorname{csch}^{2}(c x)$ to get

$$
-\frac{\log ^{k}(a) \operatorname{csch}^{2}(c x)}{2 \Gamma(k+1)}=-\frac{1}{4 \pi i} \int_{C} a^{w} w^{-k-1} \operatorname{csch}^{2}(c x) d w
$$

Deriving the second contour we use equation (2) and replace $y$ by $\log (a)-2 x$ and multiply by $\frac{1}{8} e^{-2 m x} \operatorname{csch}^{2}(c x)$ to get

$$
\frac{e^{-2 m x} \operatorname{csch}^{2}(c x)(\log (a)-2 x)^{k}}{8 \Gamma(k+1)}=\frac{1}{16 \pi i} \int_{C} a^{w} w^{-k-1} \operatorname{csch}^{2}(c x) e^{-2 x(m+w)} d w
$$

Deriving the third contour we use equation (2) and replace $y$ by $\log (a)+2 x$ and multiply by $\frac{1}{4} e^{2 m x} \operatorname{csch}^{2}(c x)$ to get

$$
\frac{e^{2 m x} \operatorname{csch}^{2}(c x)(\log (a)+2 x)^{k}}{4 \Gamma(k+1)}=\frac{1}{8 \pi i} \int_{C} a^{w} w^{-k-1} \operatorname{csch}^{2}(c x) e^{2 x(m+w)} d w
$$

Next we add equations (3), (4) and (5) then take the infinite integral over $x \in[0, \infty)$ to get

$$
\frac{1}{\Gamma(k+1)} \int_{0}^{\infty} \operatorname{csch}^{2}(c x)\left(e^{-2 m x}(\log (a)-2 x)^{k}+e^{2 m x}(\log (a)+2 x)^{k}-2 \log ^{k}(a)\right) d x
$$




$$
\begin{gathered}
=\frac{1}{2 \pi i} \int_{0}^{\infty} \int_{C} a^{w} w^{-k-1} \operatorname{csch}^{2}(c x) \sinh ^{2}(x(m+w)) d w d x \\
=\frac{1}{2 \pi i} \int_{C} \int_{0}^{\infty} a^{w} w^{-k-1} \operatorname{csch}^{2}(c x) \sinh ^{2}(x(m+w)) d x d w \\
=\frac{1}{2 \pi i} \int_{C} \frac{a^{w} w^{-k-1}\left(c-\pi(m+w) \cot \left(\frac{\pi(m+w)}{c}\right)\right)}{2 c^{2}} d w
\end{gathered}
$$

from equation (2.4.4.2) in [12] where $z=\frac{m+w}{c},-1<\operatorname{Re}(z)<1$ and $\operatorname{Im}(z)>0$, $|R e(c)|>m$. We are able to switch the order of integration over $w$ and $x$, using Fubini's theorem since the integrand is of bounded measure over the space $C \times[0, \infty)$.

\section{The Lerch function}

We use (9.550) and (9.556) in [8] where $\Phi(z, s, v)$ is the Lerch function which is a generalization of the Hurwitz zeta $\zeta(s, v)$ and Polylogarithm functions $L i_{n}(z)$. The Lerch function has a series representation given by

$$
\Phi(z, s, v)=\sum_{n=0}^{\infty}(v+n)^{-s} z^{n}
$$

where $|z|<1, v \neq 0,-1, .$. and is continued analytically by its integral representation given by

$$
\Phi(z, s, v)=\frac{1}{\Gamma(s)} \int_{0}^{\infty} \frac{t^{s-1} e^{-v t}}{1-z e^{-t}} d t=\frac{1}{\Gamma(s)} \int_{0}^{\infty} \frac{t^{s-1} e^{-(v-1) t}}{e^{t}-z} d t
$$

where $\operatorname{Re}(v)>0$, and either $|z| \leq 1, z \neq 1, \operatorname{Re}(s)>0$, or $z=1, \operatorname{Re}(s)>1$.

\subsection{Infinite sum of the first contour integral}

In this section we will again use Cauchy's integral formula (2) and taking the infinite sum to derive equivalent sum representations for the contour integrals. We proceed using equation $(2)$ and replace $y$ by $\log (a)+\frac{2 i \pi(y+1)}{c}$ and multiply both sides by $\frac{i \pi m}{c^{2}} e^{\frac{2 i \pi m(y+1)}{c}}$ and simplifying to get

$$
\begin{aligned}
& \frac{\pi^{k+1} m\left(\frac{i}{c}\right)^{k+1} e^{\frac{2 i \pi m(y+1)}{c}}\left(-\frac{i c \log (a)}{\pi}+2 y+2\right)^{k}}{c \Gamma(k+1)} \\
&=\frac{1}{2 \pi i} \int_{C} \frac{i \pi m a^{w} w^{-k-1} e^{\frac{2 i \pi(y+1)(m+w)}{c}}}{c^{2}} d w
\end{aligned}
$$

Next we take the infinite sum over $y \in[0, \infty)$ and simplify using the Lerch function to get 


$$
\begin{aligned}
& \frac{2^{k} \pi^{k+1} m\left(\frac{i}{c}\right)^{k+1} e^{\frac{2 i \pi m}{c}} \Phi\left(e^{\frac{2 i m \pi}{c}},-k, 1-\frac{i c \log (a)}{2 \pi}\right)}{c \Gamma(k+1)} \\
& =\frac{1}{2 \pi i} \sum_{y=0}^{\infty} \int_{C} \frac{i \pi m a^{w} w^{-k-1} e^{\frac{2 i \pi(y+1)(m+w)}{c}}}{c^{2}} d w \\
& =\frac{1}{2 \pi i} \int_{C} \sum_{y=0}^{\infty} \frac{i \pi m a^{w} w^{-k-1} e^{\frac{2 i \pi(y+1)(m+w)}{c}}}{c^{2}} d w \\
& =-\frac{1}{2 \pi i} \int_{C} \frac{\pi m a^{w} w^{-k-1}\left(\cot \left(\frac{\pi(m+w)}{c}\right)+i\right)}{2 c^{2}} d w
\end{aligned}
$$

from (1.232.1) in [8] and $\operatorname{Im}(w+m)>0$ for convergence of the sum.

\subsection{Infinite sum of the second contour integral}

The derivation in this section is equivalent to Section (2.1) after multiplication by $m$ and replacement of $k \rightarrow(k-1)$.

$$
\begin{aligned}
& \frac{2^{k-1} \pi^{k}\left(\frac{i}{c}\right)^{k} e^{\frac{2 i \pi m}{c}} \Phi\left(e^{\frac{2 i m \pi}{c}}, 1-k, 1-\frac{i c \log (a)}{2 \pi}\right)}{c(k-1) !} \\
&=-\frac{1}{2 \pi i} \int_{C} \frac{\pi a^{w} w^{-k}\left(\cot \left(\frac{\pi(m+w)}{c}\right)+i\right)}{2 c^{2}} d w
\end{aligned}
$$

from (1.232.1) in [8] and $\operatorname{Im}(w+m)>0$ for convergence of the sum.

\subsection{Additional contours}

In this section we will derive the additional contours from equations (6), (10) and (11). We proceed using equation (2) and replace $y$ by $\log (a)$ and multiply both sides by $\frac{1}{2 c}$ and simplifying to get

$$
\frac{\log ^{k}(a)}{2 c \Gamma(k+1)}=\frac{1}{2 \pi i} \int_{C} \frac{a^{w} w^{-k-1}}{2 c} d w
$$

Next using equation (2) and replace $y$ by $\log (a)$ and multiply both sides by $-\frac{i \pi m}{2 c^{2}}$ and simplifying to get

$$
\frac{i \pi m \log ^{k}(a)}{2 c^{2} \Gamma(k+1)}=-\frac{1}{2 \pi i} \int_{C} \frac{i \pi m a^{w} w^{-k-1}}{2 c^{2}} d w
$$

Next using equation (2) and replace $y$ by $\log (a), k$ by $k-1$ and multiply both sides by $-\frac{i \pi}{2 c^{2}}$ and simplifying to get 


$$
-\frac{i \pi \log ^{k-1}(a)}{2 c^{2} \Gamma(k)}=-\frac{1}{2 \pi i} \int_{C} \frac{i \pi a^{w} w^{-k}}{2 c^{2}} d w
$$

Note that the equations (12), (13) and (14) are equivalent to (3) with $\operatorname{csch}(c x)$ dropped and different multipliers.

\section{Definite integral in terms of the Lerch function}

Theorem 1. For all $k, a \in \mathbb{C},|\operatorname{Re}(c)|>m$,

$$
\begin{aligned}
\int_{0}^{\infty} \operatorname{csch}^{2}(c x)\left(e^{-2 m x}(\log (a)-2 x)^{k}+e^{2 m x}(\log (a)+2 x)^{k}-2 \log ^{k}(a)\right) d x \\
=\frac{i 2^{k+2} \pi^{k+1} m\left(\frac{i}{c}\right)^{k} e^{\frac{2 i \pi m}{c}} \Phi\left(e^{\frac{2 i m \pi}{c}},-k, 1-\frac{i c \log (a)}{2 \pi}\right)}{c^{2}}+\frac{2 i \pi m \log ^{k}(a)}{c^{2}} \\
+\frac{2 i \pi k \log ^{k-1}(a)}{c^{2}}+\frac{2^{k+1} k \pi^{k}\left(\frac{i}{c}\right)^{k} e^{\frac{2 i \pi m}{c}} \Phi\left(e^{\frac{2 i m \pi}{c}}, 1-k, 1-\frac{i c \log (a)}{2 \pi}\right)}{c} \\
+\frac{2 \log ^{k}(a)}{c}
\end{aligned}
$$

Proof. Since the right-hand side of equation (6) is equal to the sum of the right-hand sides of equations (10), (11), (12), (13) and (14), we can equate the left-hand sides to achieve the stated result.

\section{Derivation of entry $(2.4 .4 .2)$ in $[12]$}

Corollary 1. For $|\operatorname{Re}(c)|>m$,

$$
\int_{0}^{\infty} \operatorname{csch}^{2}(c x) \sinh ^{2}(m x) d x=\frac{c-\pi m \cot \left(\frac{\pi m}{c}\right)}{2 c^{2}}
$$

Proof. Use equation (15) and set $k=0$ and simplify using entry (2) in Table below $(64: 12: 7)$ in [11].

\section{Derivation of new entry for Table 2.4.4 in [12]}

Corollary 2. For all $\operatorname{Re}(\beta)>|\operatorname{Re}(\alpha)| / 2$,

$$
\int_{0}^{\infty} x \sinh (\alpha x) \operatorname{csch}^{2}(\beta x) d x=\frac{\pi\left(\pi \alpha-\beta \sin \left(\frac{\pi \alpha}{\beta}\right)\right) \csc ^{2}\left(\frac{\pi \alpha}{2 \beta}\right)}{4 \beta^{3}}
$$

Proof. Use equation (15) and set $k=1, a=1, m=\alpha / 2, c=\beta$ and simplify using entry (1) in Table below (64:12:7) in [11]. 


\section{Derivation of the Mellin transform}

In this section we will derive a generalize Mellin transform of equations (2.3.1.19) in [7], (3.527.8) and (3.527.9) in [8].

Theorem 2. For all $\operatorname{Re}(s)>0, \operatorname{Re}(\beta)=\sqrt{|\operatorname{Re}(\alpha)|}$,

$$
\begin{aligned}
\int_{0}^{\infty} & x^{s-1} \cosh (\alpha x) \operatorname{csch}^{2}(\beta x) d x \\
= & -\frac{1}{2} \pi^{s-1}\left(\frac{1}{\beta}\right)^{s} \csc \left(\frac{\pi s}{2}\right) L i_{2-s}\left(e^{-\frac{i \pi \alpha}{\beta}}\right)+\frac{1}{2} \pi^{s-1} s\left(\frac{1}{\beta}\right)^{s} \csc \left(\frac{\pi s}{2}\right) L i_{2-s}\left(e^{-\frac{i \pi \alpha}{\beta}}\right) \\
& -\frac{1}{2} \pi^{s-1}\left(\frac{1}{\beta}\right)^{s} \csc \left(\frac{\pi s}{2}\right) L i_{2-s}\left(e^{\frac{i \pi \alpha}{\beta}}\right)+\frac{1}{2} \pi^{s-1} s\left(\frac{1}{\beta}\right)^{s} \csc \left(\frac{\pi s}{2}\right) L i_{2-s}\left(e^{\frac{i \pi \alpha}{\beta}}\right) \\
- & \frac{1}{2} i \alpha \pi^{s}\left(\frac{1}{\beta}\right)^{s+1} \csc \left(\frac{\pi s}{2}\right) L i_{1-s}\left(e^{-\frac{i \pi \alpha}{\beta}}\right)+\frac{1}{2} i \alpha \pi^{s}\left(\frac{1}{\beta}\right)^{s+1} \csc \left(\frac{\pi s}{2}\right) L i_{1-s}\left(e^{\frac{i \pi \alpha}{\beta}}\right)
\end{aligned}
$$

Proof. Use equation (15) set $m=m / 2, a=e^{2 a}$ and replace $m$ by $-m$ to form a second equation and take their difference to get

$$
\begin{aligned}
\int_{0}^{\infty}\left((a-x)^{k}-(a+x)^{k}\right) \operatorname{csch}^{2}(c x) \sinh (m x) d x & \\
=-\frac{i \pi m a^{k}}{c^{2}}-\frac{i \pi^{k+1} m\left(\frac{i}{c}\right)^{k} e^{-\frac{i \pi m}{c}} \Phi\left(e^{-\frac{i m \pi}{c}},-k, 1-\frac{i a c}{\pi}\right)}{c^{2}} & \\
-\frac{i \pi^{k+1} m\left(\frac{i}{c}\right)^{k} e^{\frac{i \pi m}{c}} \Phi\left(e^{\frac{i m \pi}{c}},-k, 1-\frac{i a c}{\pi}\right)}{c^{2}}+\frac{k \pi^{k}\left(\frac{i}{c}\right)^{k} e^{-\frac{i \pi m}{c}} \Phi\left(e^{-\frac{i m \pi}{c}}, 1-k, 1-\frac{i a c}{\pi}\right)}{c} & -\frac{k \pi^{k}\left(\frac{i}{c}\right)^{k} e^{\frac{i \pi m}{c}} \Phi\left(e^{\frac{i m \pi}{c}}, 1-k, 1-\frac{i a c}{\pi}\right)}{c}
\end{aligned}
$$

Next set $a=0, m=\alpha, c=\beta, k=s-1$ and simplify using equation (64:12:2) in [11].

\section{Derivation of (3.527.1) in $[8]$}

Theorem 3. For all $\operatorname{Re}(a)>0, \operatorname{Re}(\mu)>2$,

$$
\int_{0}^{\infty} x^{\mu-1} \operatorname{csch}^{2}(a x) d x=2^{2-\mu}\left(\frac{1}{a}\right)^{\mu} \Gamma(\mu) \zeta(\mu-1)
$$

Proof. Use equation (18) and set $\alpha=0, s=\mu, \beta=a$ and simplify using equation $(25: 12: 5)$ in [11]. 


\section{Derivation of entry (3.527.2) in [8]}

Theorem 4. For all $\operatorname{Re}(\beta)>0$,

$$
\int_{0}^{\infty} x^{2 m} \operatorname{csch}^{2}(\beta x) d x=\pi^{2 m}\left(\frac{1}{\beta}\right)^{2 m+1}\left|B_{2 m}\right|
$$

Proof. Use equation (20) and set $\mu=2 m+1$ and simplify using equation (3:13:1) in [11].

\section{Derivation of entry (3.527.9) in [8]}

Theorem 5. For all $\operatorname{Re}(m)>0, \operatorname{Re}(a)>0$,

$$
\begin{aligned}
& \int_{0}^{\infty} x^{2 m+1} \operatorname{coth}(a x) \operatorname{csch}(a x) d x \\
&=\left(1-2^{2 m+1}\right)(2 m+1) \pi^{2 m+1}\left(\frac{1}{a}\right)^{2 m+2} \zeta(-2 m) \csc (\pi m)
\end{aligned}
$$

Proof. Use equation (18) and set $s=2 m+2, \alpha=\beta=a$ and simplify using entry (4) in Table below $(25: 12: 5)$ in [11].

\section{Derivation of entry $(3.527 .10)$ in [8]}

Corollary 3. For all $\operatorname{Re}(a)>0, \operatorname{Re}(m)>1 / 2$,

$$
\int_{0}^{\infty} x^{2 m} \operatorname{coth}(a x) \operatorname{csch}(a x) d x=4^{1-m}\left(4^{m}-1\right) m\left(\frac{1}{a}\right)^{2 m+1} \zeta(2 m) \Gamma(2 m)
$$

Proof. Use equation (18) and set $s=2 m+1, \alpha=a, \beta=a$ and simplify using entry (4) in Table below $(3: 13: 1)$ in [11].

\section{Derivation of entry (3.527.12) in [8]}

Corollary 4.

$$
\int_{0}^{\infty} x^{2} \operatorname{csch}^{2}(x) d x=\frac{\pi^{2}}{6}
$$

Proof. Use equation (21) and set $m=\beta=1$ and simplify. 


\section{Derivation of entry (3.527.13) in [8]}

Corollary 5. For all $\operatorname{Re}(a)>0$

$$
\int_{0}^{\infty} x^{2} \operatorname{coth}(a x) \operatorname{csch}(a x) d x=\frac{\pi^{2}}{2 a^{3}}
$$

Proof. Use equation (18) and set $s=3, \alpha=\beta=a$ and simplify.

\section{Derivation of entry (3.527.16) in [8] and (2.4.5.12) in [12]}

Theorem 6. For all $\operatorname{Re}(a)>0, \operatorname{Re}(\mu)>2$

$$
\int_{0}^{\infty} x^{\mu-1} \operatorname{coth}(a x) \operatorname{csch}(a x) d x=2^{1-\mu}\left(2^{\mu}-2\right)\left(\frac{1}{a}\right)^{\mu} \Gamma(\mu) \zeta(\mu-1)
$$

Proof. Use equation (18) and set $s=\mu, \alpha=\beta=a$ and simplify using entry (4) in Table below $(25: 12: 5)$ in [11].

\section{Derivation of entry (2.3.1.9) in Brychkov, (3.523.1) in [8]}

Theorem 7. For all $\operatorname{Re}(s)>1, \operatorname{Re}(\alpha)>0$

$$
\int_{0}^{\infty} x^{s-1} \operatorname{csch}(\alpha x) d x=2^{1-s}\left(2^{s}-1\right)\left(\frac{1}{\alpha}\right)^{s} \zeta(s) \Gamma(s)
$$

Proof. Use equation (18) and take the first partial derivative with respect to $\alpha$ then set $\alpha=\beta, s=s-1$ and simplify using entry (4) in Table below (25:12:5) in [11].

\section{Derivation of entry (3.523.2) in [8]}

Theorem 8. For all $\operatorname{Re}(a)>0, n=1,2,$. .

$$
\int_{0}^{\infty} x^{2 n-1} \operatorname{csch}(\alpha x) d x=\frac{\left(4^{n}-1\right) \pi^{2 n}\left(\frac{1}{\alpha}\right)^{2 n}\left|B_{2 n}\right|}{2 n}
$$

Proof. Use equation (18) and take the first partial derivative with respect to $\alpha$ then set $\alpha=\beta, s=2 n$ and simplify using equation (3:13:1) in [11].

\section{Derivation of entry (3.523.6) in [8]}

\section{Corollary 6.}

$$
\int_{0}^{\infty} x^{3} \operatorname{csch}(x) d x=\frac{\pi^{4}}{8}
$$

Proof. Use equation (18) and take the first partial derivative with respect to $\alpha$ then set $\alpha=\beta=1, s=3$ and simplify using entry (2) in Table below (25:12:5) in [11]. 


\section{Derivation of entry (3.523.8) in [8]}

\section{Corollary 7.}

$$
\int_{0}^{\infty} x^{5} \operatorname{csch}(x) d x=\frac{\pi^{6}}{4}
$$

Proof. Use equation (18) and take the first partial derivative with respect to $\alpha$ then set $\alpha=\beta=1, s=5$ and simplify using entry (2) in Table below (25:12:5) in [11].

\section{Derivation of entry $(3.523 .10)$ in $[8]$}

\section{Corollary 8.}

$$
\int_{0}^{\infty} x^{7} \operatorname{csch}(x) d x=\frac{17 \pi^{8}}{16}
$$

Proof. Use equation (18) and take the first partial derivative with respect to $\alpha$ then set $\alpha=\beta=1, s=7$ and simplify using entry (2) in Table below (25:12:5) in [11].

\section{Derivation of entry (3.521.1) in [8]}

Theorem 9. For $\alpha \in \mathbb{C}$,

$$
\int_{0}^{\infty} x \operatorname{csch}(\alpha x) d x= \begin{cases}\frac{\pi^{2}}{4 \alpha^{2}}, & \text { for } \operatorname{Re}(\alpha)>0 \\ -\frac{\pi^{2}}{4 \alpha^{2}}, & \text { for } \operatorname{Re}(\alpha)<0\end{cases}
$$

Proof. Use equation (18) and take the first partial derivative with respect to $\alpha$ then set $\alpha=\beta, s=1$ and simplify using entry (2) in Table below (25:12:5) in [11].

\section{Derivations in terms of Catalan's constant $C$ and $\pi$}

\section{Corollary 9.}

$$
\int_{0}^{\infty} \frac{\operatorname{csch}(x)\left(-4 x^{2} \operatorname{csch}(x)+\pi^{2} \operatorname{coth}(x)-\pi^{2} \operatorname{csch}(x)\right)}{4 x^{2}+\pi^{2}} d x=-2(C-1)
$$

Corollary 10.

$$
\int_{0}^{\infty} \frac{x \operatorname{csch}(x)}{4 x^{2}+\pi^{2}} d x=\frac{1}{8}(\pi-2)
$$

Proof. Use equation (15) and set $k=-1, a=-1, c=1, m=1 / 2$ and simplify in terms of Catalan's constant $C$, using entries (1) and (4) in Tables below (64:12:7) in [11] and equations (2.3) and (2.7) in [10] and equation (9.73) in [8] and simplify in terms of the real and imaginary parts. 
Corollary 11.

$$
\int_{0}^{\infty} \frac{x \operatorname{csch}(x)}{x^{2}+\pi^{2}} d x=\log (2)-\frac{1}{2}
$$

Corollary 12.

$$
\int_{0}^{\infty} \frac{x \sinh (x) \operatorname{csch}^{2}(2 x)}{x^{2}+\pi^{2}} d x=\frac{8 C-8+\pi(\log (4)-1)}{8 \pi}
$$

Proof. Use equation (19) and set $k=-1, a=\pi i, m=1, c=1$ and $k=-1, a=\pi i, m=$ $1, c=2$ respectively and simplify using entries (1) and (4) in Tables below (64:12:7) in [11] and equations (2.3) and (2.7) in [10].

\section{Derivation of a new entry for Table 2.4.5 in [12]}

Theorem 10. For all $\operatorname{Re}(c)>0, \operatorname{Re}(m)>0, z \in \mathbb{C}$,

$$
\begin{aligned}
& \int_{0}^{\infty} \frac{x \operatorname{csch}^{2}(c x) \sinh (m x)}{x^{2}+z^{2}} d x \\
&=\frac{\pi m}{2 c^{2} z}+\frac{m e^{-\frac{i \pi m}{c}} \Phi\left(e^{-\frac{i m \pi}{c}}, 1, \frac{c z}{\pi}+1\right)}{2 c}+\frac{m e^{\frac{i \pi m}{c}} \Phi\left(e^{\frac{i m \pi}{c}}, 1, \frac{c z}{\pi}+1\right)}{2 c} \\
&-\frac{i e^{-\frac{i \pi m}{c}} \Phi\left(e^{-\frac{i m \pi}{c}}, 2, \frac{c z}{\pi}+1\right)}{2 \pi}+\frac{i e^{\frac{i \pi m}{c}} \Phi\left(e^{\frac{i m \pi}{c}}, 2, \frac{c z}{\pi}+1\right)}{2 \pi}
\end{aligned}
$$

Proof. Use equation (19) and set $k=-1, a=z i$ and simplify.

\section{Derivations in terms of Catalan's constant $C$ and $\pi$}

Theorem 11. For all $k, a \in \mathbb{C}, \operatorname{Re}(c)>0$,

$$
\begin{array}{r}
\int_{0}^{\infty} 2 \operatorname{csch}^{2}(c x)\left(\cosh (2 m x)\left((\log (a)-2 x)^{k}+(\log (a)+2 x)^{k}\right)-2 \log ^{k}(a)\right) d x \\
=-\frac{i 2^{k+2} \pi^{k+1} m\left(\frac{i}{c}\right)^{k} e^{-\frac{2 i \pi m}{c}} \Phi\left(e^{-\frac{2 i m \pi}{c}},-k, 1-\frac{i c \log (a)}{2 \pi}\right)}{c^{2}} \\
+\frac{i 2^{k+2} \pi^{k+1} m\left(\frac{i}{c}\right)^{k} e^{\frac{2 i \pi m}{c}} \Phi\left(e^{\frac{2 i m \pi}{c}},-k, 1-\frac{i c \log (a)}{2 \pi}\right)}{c^{2}} \\
+\frac{4 i \pi k \log ^{k-1}(a)}{c^{2}}+\frac{2^{k+1} k \pi^{k}\left(\frac{i}{c}\right)^{k} e^{-\frac{2 i \pi m}{c}} \Phi\left(e^{-\frac{2 i m \pi}{c}}, 1-k, 1-\frac{i c \log (a)}{2 \pi}\right)}{c} \\
+\frac{2^{k+1} k \pi^{k}\left(\frac{i}{c}\right)^{k} e^{\frac{2 i \pi m}{c}} \Phi\left(e^{\frac{2 i m \pi}{c}}, 1-k, 1-\frac{i c \log (a)}{2 \pi}\right)}{c}+\frac{4 \log ^{k}(a)}{c}
\end{array}
$$


Proof. Use equation (18) and form a second equation by replacing $m$ by $-m$ and adding both and simplify.

\section{Corollary 13.}

$$
\int_{0}^{\infty} \frac{\left(-4 x^{2}+\pi^{2} \cosh (x)-\pi^{2}\right) \operatorname{csch}^{2}(x)}{2\left(4 x^{2}+\pi^{2}\right)} d x=1-C
$$

Proof. Use equation (38) and set $k=-1, a=-1, m=1 / 2, c=1$ and simplify using entries (1) and (4) in Tables below (64:12:7) in [11] and equations (2.3) and (2.7) in [10] and equation (9.73) in [8].

\section{Corollary 14.}

$$
\int_{0}^{\infty} \frac{\left(-4 x^{2}+\pi^{2} \cosh (x)-\pi^{2}\right) \operatorname{csch}^{2}(2 x)}{4 x^{2}+\pi^{2}} d x=\frac{1}{8}(-4 C+6-\pi \log (2))
$$

Proof. Use equation (38) and set $k=-1, a=-1, m=1 / 2, c=2$ and simplify using entries (1) and (4) in Tables below (64:12:7) in [11] and equations (2.3) and (2.7) in [10] and equation (9.73) in [8].

\section{Definite integral involving the arctangent function in terms of the log-gamma and Harmonic number functions}

Theorem 12. For all $a \in \mathbb{C}, \operatorname{Re}(c)>0$,

$$
\begin{aligned}
& \int_{0}^{\infty} x \tanh ^{-1}\left(\frac{x}{a}\right) \operatorname{coth}(c x) \operatorname{csch}(c x) d x \\
& =\frac{a c\left(H_{-\frac{i a c}{2 \pi}}-H_{-\frac{i a c+\pi}{2 \pi}}\right)+i \pi\left(-1+2 \log \left(\frac{i(-\pi-i a c) \Gamma\left(-\frac{i a c+\pi}{2 \pi}\right)}{\sqrt{2 \pi} \sqrt{a} \sqrt{\frac{i}{c}} c \Gamma\left(-\frac{i a c}{2 \pi}\right)}\right)\right)}{2 c^{2}}
\end{aligned}
$$

Proof. Use equation (19) and take the first partial derivative with respect to $m$. Next set $m=c$, followed by taking the first partial derivative with respect to $k$ then applying L'Hopital's rule as $k \rightarrow 0$ and simplify using equations (64:10:2), (64:4:1), (44:1:1) and entry (4) in Table below (64:12:7) in [11].

\section{Corollary 15.}

$$
\begin{aligned}
& \int_{0}^{\infty} x \tanh ^{-1}(x) \operatorname{coth}(x) \operatorname{csch}(x) d x \\
&=\frac{1}{2} H_{-\frac{i}{2 \pi}}-\frac{1}{2} H_{-\frac{i+\pi}{2 \pi}}-\frac{i \pi}{2}+\frac{3 \pi^{2}}{4}-\frac{1}{2} i \pi \log \left(\frac{2 \pi}{(\pi+i)^{2}}\right) \\
& \quad-i \pi \log \left(\Gamma\left(-\frac{i}{2 \pi}\right)\right)+i \pi \log \left(\Gamma\left(-\frac{i+\pi}{2 \pi}\right)\right)
\end{aligned}
$$


Proof. Use equation (41) and set $a=c=1$ and simplify. Note: There exists a singularity at $x=1$.

Corollary 16. For $a \in \mathbb{C}$,

$\int_{0}^{\infty} x \cot \left(\frac{\pi x}{2 a}\right) \csc \left(\frac{\pi x}{2 a}\right) \tanh ^{-1}\left(\frac{x}{a}\right) d x= \begin{cases}-\frac{i a^{2}}{\pi}\left(-2+\pi+\log \left(\frac{16 \Gamma\left(\frac{1}{4}\right)^{4}}{\Gamma\left(-\frac{1}{4}\right)^{4}}\right)\right), & \text { for } \operatorname{Im}(a)>0 \\ \frac{i a^{2}}{\pi}\left(-2+\pi+\log \left(\frac{16 \Gamma\left(\frac{1}{4}\right)^{4}}{\Gamma\left(-\frac{1}{4}\right)^{4}}\right)\right), & \text { for } \operatorname{Im}(a)<0\end{cases}$

Proof. Use equation (41) and set $c=\frac{i \pi}{2 a}$ and simplify.

\section{Corollary 17.}

$$
\int_{0}^{\infty} x \tan ^{-1}(x) \operatorname{coth}\left(\frac{\pi x}{2}\right) \operatorname{csch}\left(\frac{\pi x}{2}\right) d x=\frac{-2+\pi+\log \left(\frac{16 \Gamma\left(\frac{1}{4}\right)^{4}}{\Gamma\left(-\frac{1}{4}\right)^{4}}\right)}{\pi}
$$

Proof. Use equation (43) and set $a=i$.

\section{Corollary 18.}

$$
\int_{0}^{\infty} x \tan ^{-1}\left(\frac{x}{\sqrt{\pi}}\right) \operatorname{coth}\left(\frac{\sqrt{\pi} x}{2}\right) \operatorname{csch}\left(\frac{\sqrt{\pi} x}{2}\right) d x=-2+\pi+\log \left(\frac{16 \Gamma\left(\frac{1}{4}\right)^{4}}{\Gamma\left(-\frac{1}{4}\right)^{4}}\right)
$$

Proof. Use equation (43) and set $a=\sqrt{2} i$.

Corollary 19. For $a \in \mathbb{C}$,

$$
\begin{aligned}
& \int_{0}^{\infty} x \cot \left(\frac{\pi x}{a}\right) \\
& \csc \left(\frac{\pi x}{a}\right) \\
& \tanh ^{-1}\left(\frac{x}{a}\right) d x= \begin{cases}-\frac{i a^{2}}{2 \pi}(-1+\log (2)+\log (\pi)), & \text { for } \operatorname{Im}(a)>0 \\
\frac{i a^{2}}{2 \pi}(-1+\log (2)+\log (\pi)), & \text { for } \operatorname{Im}(a)<0\end{cases}
\end{aligned}
$$

Proof. Use equation (41) and apply L'Hopital's rule as $c \rightarrow \frac{i \pi}{a}$ and simplify.

\section{Corollary 20.}

$$
\int_{0}^{\infty} x \tan ^{-1}(x) \operatorname{coth}(\pi x) \operatorname{csch}(\pi x) d x=\frac{\log (2 \pi)-1}{2 \pi}
$$


R. Reynolds, A. Stauffer / Eur. J. Pure Appl. Math, 14 (4) (2021), 1132-1147

Proof. Use equation (46) and set $a=i$.

\section{Corollary 21.}

$$
\int_{0}^{\infty} x \tan ^{-1}\left(\frac{x}{\sqrt{2 \pi}}\right) \operatorname{coth}\left(\sqrt{\frac{\pi}{2}} x\right) \operatorname{csch}\left(\sqrt{\frac{\pi}{2}} x\right) d x=\log (2 \pi)-1
$$

Proof. Use equation (46) and set $a=\sqrt{2 \pi} i$. 


\section{Tables of results}

Table 1: Table of definite integrals

\begin{tabular}{|c|c|}
\hline$f(x)$ & $\int_{0}^{\infty} f(x) d x$ \\
\hline $\operatorname{csch}^{2}(c x) \sinh ^{2}(m x)$ & $\frac{c-\pi m \cot \left(\frac{\pi m}{c}\right)}{2 c^{2}}$ \\
\hline$x \sinh (\alpha x) \operatorname{csch}^{2}(\beta x)$ & $\frac{\pi\left(\pi \alpha-\beta \sin \left(\frac{\pi \alpha}{\beta}\right)\right) \csc ^{2}\left(\frac{\pi \alpha}{2 \beta}\right)}{4 \beta^{3}}$ \\
\hline$x^{\mu-1} \operatorname{csch}^{2}(a x)$ & $2^{2-\mu}\left(\frac{1}{a}\right)^{\mu} \Gamma(\mu) \zeta(\mu-1)$ \\
\hline$x^{2 m} \operatorname{csch}^{2}(\beta x)$ & $\pi^{2 m}\left(\frac{1}{\beta}\right)^{2 m+1}\left|B_{2 m}\right|$ \\
\hline$x^{2 m} \operatorname{coth}(a x) \operatorname{csch}(a x)$ & $4^{1-m}\left(4^{m}-1\right) m\left(\frac{1}{a}\right)^{2 m+1} \zeta(2 m) \Gamma(2 m)$ \\
\hline$x^{2} \operatorname{csch}^{2}(x)$ & $\frac{\pi^{2}}{6}$ \\
\hline$x^{2} \operatorname{coth}(a x) \operatorname{csch}(a x)$ & $\frac{\pi^{2}}{2 a^{3}}$ \\
\hline$x^{\mu-1} \operatorname{coth}(a x) \operatorname{csch}(a x)$ & $2^{1-\mu}\left(2^{\mu}-2\right)\left(\frac{1}{a}\right)^{\mu} \Gamma(\mu) \zeta(\mu-1)$ \\
\hline$x^{s-1} \operatorname{csch}(\alpha x)$ & $2^{1-s}\left(2^{s}-1\right)\left(\frac{1}{\alpha}\right)^{s} \zeta(s) \Gamma(s)$ \\
\hline$x^{2 n-1} \operatorname{csch}(\alpha x)$ & $\frac{\left(4^{n}-1\right) \pi^{2 n}\left(\frac{1}{\alpha}\right)^{2 n}\left|B_{2 n}\right|}{2 n}$ \\
\hline$x^{3} \operatorname{csch}(x)$ & $\frac{\pi^{4}}{8}$ \\
\hline$x^{5} \operatorname{csch}(x)$ & $\frac{\pi^{6}}{4}$ \\
\hline$x^{7} \operatorname{csch}(x)$ & $\frac{17 \pi^{8}}{16}$ \\
\hline$x \operatorname{csch}(\alpha x)$ & $\frac{\pi^{2}}{4 \alpha^{2}}$ \\
\hline$\frac{\operatorname{csch}(x)\left(-4 x^{2} \operatorname{csch}(x)+\pi^{2} \operatorname{coth}(x)-\pi^{2} \operatorname{csch}(x)\right)}{4 x^{2}+\pi^{2}}$ & $-2(C-1)$ \\
\hline
\end{tabular}




\begin{tabular}{lc}
\hline$f(x)$ & $\int_{0}^{\infty} f(x) d x$ \\
\hline$\frac{x \operatorname{csch}(x)}{4 x^{2}+\pi^{2}}$ & $\frac{1}{8}(\pi-2)$ \\
$\frac{x \operatorname{csch}(x)}{x^{2}+\pi^{2}}$ & $\log (2)-\frac{1}{2}$ \\
$\frac{x \sinh (x) \operatorname{csch}^{2}(2 x)}{x^{2}+\pi^{2}}$ & $\frac{8 C-8+\pi(\log (4)-1)}{8 \pi}$ \\
$\frac{\left(-4 x^{2}+\pi^{2} \cosh (x)-\pi^{2}\right) \operatorname{csch}^{2}(x)}{2\left(4 x^{2}+\pi^{2}\right)}$ & $\frac{1-C}{8}(-4 C+6-\pi \log (2))$ \\
$\frac{\left(-4 x^{2}+\pi^{2} \cosh (x)-\pi^{2}\right) \operatorname{csch}^{2}(2 x)}{4 x^{2}+\pi^{2}}$ & $-2+\pi+\log \left(\frac{16 \Gamma\left(\frac{1}{4}\right)^{4}}{\Gamma\left(-\frac{1}{4}\right)^{4}}\right)$ \\
& $\frac{\pi}{\pi}$ \\
$x \tan ^{-1}(x) \operatorname{coth}\left(\frac{\pi x}{2}\right) \operatorname{csch}\left(\frac{\pi x}{2}\right)$ & $-2+\pi+\log \left(\frac{16 \Gamma\left(\frac{1}{4}\right)^{4}}{\Gamma\left(-\frac{1}{4}\right)^{4}}\right)$ \\
$x \tan ^{-1}(x) \operatorname{coth}\left(\frac{\pi x}{2}\right) \operatorname{csch}\left(\frac{\pi x}{2}\right)$ & $-2+\pi+\log \left(\frac{16 \Gamma\left(\frac{1}{4}\right)^{4}}{\Gamma\left(-\frac{1}{4}\right)^{4}}\right)$ \\
$x \tan ^{-1}\left(\frac{x}{\sqrt{\pi}}\right) \operatorname{coth}\left(\frac{\sqrt{\pi} x}{2}\right) \operatorname{csch}\left(\frac{\sqrt{\pi} x}{2}\right)$ & $\frac{\log (2 \pi)-1}{2 \pi}$ \\
$x \tan ^{-1}(x) \operatorname{coth}(\pi x) \operatorname{csch}(\pi x)$ & $\log (2 \pi)-1$ \\
$x \tan ^{-1}\left(\frac{x}{\sqrt{2 \pi}}\right) \operatorname{coth}\left(\sqrt{\frac{\pi}{2}} x\right) \operatorname{csch}\left(\sqrt{\frac{\pi}{2}} x\right)$ & \\
\hline
\end{tabular}

\section{Discussion}

In this work the authors derived definite integrals used in physics along with some new forms not previously published. Some of the integral forms were expressed in terms of fundamental constants such as Catalan's constant and $\pi$. The integral forms derived were achieved by the use of our contour integral method [13] and the evaluation of integrals using the Lerch function.

The Lerch function being multivariate with its analytic continuation properties allowed the authors to widen the range of computation for the formulae derived. We used Wolfram's Mathematica software for numerical evaluations for real and imaginary values of the parameters involved. Derivations of definite integrals in $[7,8,12]$ are useful as it will assist in providing formal proofs and verifying whether the formulae in these books are correct. We will be using our method to produce more tables of integrals in future work. 


\section{References}

[1] Nail Akhmediev and Adrian Ankiewicz, editors. Dissipative Solitons. Springer Berlin Heidelberg, 2005.

[2] Peter Arnold, Tyler Gorda, and Shahin Iqbal. The lpm effect in sequential bremsstrahlung: nearly complete results for qcd. Journal of High Energy Physics, 2020, 112020.

[3] A. D. Boardman and A. P. Sukhorukov. Soliton-driven Photonics. Springer Science \& Business Media, 082001.

[4] Anders Bondeson. Perturbation analysis of single langmuir solitons. Physics of Fluids, $23: 746,1980$.

[5] D. I. Borisov, G. Cardone, G. A. Chechkin, and Yu. O. Koroleva. On elliptic operators with steklov condition perturbed by dirichlet condition on a small part of boundary. Calculus of Variations and Partial Differential Equations, 60, 022021.

[6] Denis Borisov and Giuseppe Cardone. Spectra of operator pencils with small pjsymmetric periodic perturbation. ESAIM: Control, Optimisation and Calculus of Variations, 26:21, 2020.

[7] Yu A. Brychkov, O. I. Marichev, and N. V. Savischenko. Handbook of Mellin Transforms. Chapman and Hall/CRC; 1st edition (Oct. 10 2018), 102018.

[8] I. S. Gradshteyn, I. M. Ryzhik, Alan Jeffrey, and Daniel Zwillinger. Table of Integrals, Series, and Products. Academic Press; 6th edition (Aug. 24 2000), 082000.

[9] E.A. Kuznetsov, A.M. Rubenchik, and V.E. Zakharov. Soliton stability in plasmas and hydrodynamics. Physics Reports, 142:103-165, 091986.

[10] Leonard Lewin. Polylogarithms and Associated Functions. North Holland, 1981.

[11] Keith B. Oldham, Jan Myland, and Jerome Spanier. An Atlas of Functions: with Equator, the Atlas Function Calculator. Springer Science \& Business Media, 072010.

[12] Anatoliı̌ Platonovich Prudnikov, Yu A. Brychkov, and Oleg Igorevich Marichev. Integrals and Series: More special functions. Gordon and Breach Science Publishers, 1986.

[13] Robert Reynolds and Allan Stauffer. A method for evaluating definite integrals in terms of special functions with examples. International Mathematical Forum, 15:235244,2020 .

[14] Ambaresh Sahoo and Samudra Roy. Dissipative soliton mediated radiations in active silicon-based waveguides. Journal of the Optical Society of America B, 35:257, 01 2018 . 